\title{
ON THE FOURIER-STIELTJES TRANSFORM OF COMPACT OPERATOR VALUED MEASURES ASSOCIATED TO DISCRETE SERIES REPRESENTATIONS
}

\author{
KOAMI GBEMOU ${ }^{1}$, YAOGAN MENSAH ${ }^{1,2, *}$ \\ ${ }^{1}$ Department of Mathematics, University of Lomé, Togo \\ ${ }^{2}$ International Chair in Mathematical Physics and Applications (ICMPA)-Unesco Chair, University of \\ Abomey-Calavi, Benin \\ *Corresponding author: mensahyaogan2@gmail.com
}

Received Mar. 28, 2020

\begin{abstract}
Aвstract. The Fourier-Stieltjes transform of compact operators valued measures on a locally compact unimodular group is considered. The transformed measures are evaluated at a square integrable irreducible representation of the group. We obtain a decomposition of the transformed measures in terms of compact operators and an integral representation of some compact operators valued transformers on the completion of a certain tensor product space.
\end{abstract}

2020 Mathematics Subject Classification. 42A38, 48G10.

Key words and phrases. Fourier-Stieltjes transform; vector measure; locally compact group; square integrable representation; compact operator.

\section{INTRODUCTION}

The Fourier transform has shown its importance in Mathematics and its applications to Engineering, Signal processing, Physics, etc. This transform has been extended to other objects such as distributions, measures, operators...The extension to measures is known in the literature as the Fourier-Stieltjes transform.

In [3] the authors studied properties of the Fourier-Stieltjes transform of Banach space valued measures on compact groups. In [13], an interpretation of the Fourier-Stieltjes transform in terms of tensor product space was given. Other useful references are given along this paper. The aim of this paper is to pursue the study of the Fourier-Stieltjes transform of vector measures on a locally compact unimodular group. Here we are interested in $C^{*}$-algebra valued

DOI: 10.28924/APJM/7-11 
measures with particular emphasis on the $C^{*}$-algebra of compact operators. Recent works in that direction are $[11,15]$.

This article is organized as follows. Section 2 is devoted to some preliminaries. Basic facts about vector measures and group representation theory are recalled. Section 3 contents the main results.

\section{Preliminary notes}

This section is devoted to collect the necessary ingredients which we may need in the sequel. More precisely we are concerned with $C^{*}$-algebras valued measures and group representations.

2.1. $C^{*}$-algebra-valued measures. We summarize some facts about vector measures. Many assertions here are borrowed from [13]. More details about vector measures can be found in [6]. Let $\mathrm{G}$ be a locally compact group. We denote by $\mathcal{B}(G)$ the $\sigma$-field of Borel subsets of $G$. Here we set some basic facts about a general $C^{*}$-algebra valued measure though in Section 3 we will deal with a particular $C^{*}$-algebra. Let $\mathcal{A}$ be a $C^{*}$-algebra $[4,12]$. The bracket $\langle\psi, x\rangle, \psi \in \mathcal{A}^{*}, x \in \mathcal{A}$ denotes the duality between $\mathcal{A}$ and its topological dual $\mathcal{A}^{*}$. Following [6] we call a vector measure any countably additive set function $m: \mathcal{B}(G) \rightarrow \mathcal{A}$ such that for any sequence $\left(A_{n}\right)_{n \geq 1}$ of pairwise disjoint elements of $\mathcal{B}(G)$ one has

$$
m\left(\bigcup_{n=1}^{\infty} A_{n}\right)=\sum_{n=1}^{\infty} m\left(A_{n}\right) .
$$

For a vector measure $m$, the measure defined by

$$
\psi m(A):=\langle\psi, m(A)\rangle, A \in \mathcal{B}(G)
$$

where $\psi \in \mathcal{A}^{*}$, is a complex measure. The $m$-integrability of a complex Borel-measurable function $f$ defined on $\mathrm{G}$ is given by the following conditions:

1- $\forall \psi \in \mathcal{A}^{*}, f$ is $\psi m$-integrable

2- $\forall A \in \mathcal{B}(G), \exists y \in \mathcal{A}, \forall \psi \in \mathcal{A}^{*},\langle\psi, y\rangle=\int_{A} f d \psi m$.

Then we set $y=\int_{G} f d m$.

An $\mathcal{A}$-valued function $f$ defined on $G$ is $m$-integrable if the function $x \mapsto\|f(x)\|$ is $m$-integrable. A vector measure $m$ is said to be bounded if there exists $M>0$ such that

$$
\forall A \in \mathcal{B}(G),\|m(A)\| \leq M
$$


The variation of $\psi m$ is defined as

$$
|\psi m|(A)=\sup _{\pi} \sum_{n}\left|\psi m\left(A_{n}\right)\right|
$$

where the supremum is taken over all the partitions $\pi$ of $A$ into finite number of pairwise disjoint measurable subsets of $\mathcal{B}(G)$ [6, page 2]. The semivariation of a vector measure $m$ is the nonnegative set function $|m|$ defined on $\mathcal{B}(G)$ by

$$
|m|(A)=\sup \left\{|\psi m|(A): \psi \in \mathcal{A}^{*},\|\psi\| \leq 1\right\}, \forall A \in \mathcal{B}(G)
$$

where the supremum is taken over all the partitions $\pi$ of $A$ into pairwise disjoint measurable subsets of $A$. If $|m|(G)<\infty$ then $m$ is called a vector measure of bounded semivariation. Indeed, a vector measure is bounded if and only if it is of bounded semivariation [6, page 4].

Let $\mathcal{M}(G, \mathcal{A})$ denote the space of bounded $\mathcal{A}$-valued measures on $G$. We define the norm of a measure $m \in \mathcal{M}(G, \mathcal{A})$ by:

$$
\|m\|:=\int_{G} \chi_{G} d|m|=|m|(G) .
$$

2.2. Basic group representations theory and Haar measure. Here we are interested in the theory of group representations. Interested readers are refered to [2,5,8-10]. Its applications to wavelets analysis can be found in $[1,9]$.

A unitary representation of a locally compact group $G$ is a group homomorphism $\sigma: G \mapsto \mathcal{U}\left(H_{\sigma}\right)$ where $\mathcal{U}\left(H_{\sigma}\right)$ is the group of unitary operators on the complex Hilbert space $H_{\sigma}$. The space $H_{\sigma}$ is called the representation space of $\sigma$ and its dimension is called the dimension of $\sigma$. A unitary representation is said to be continuous if for any $\alpha \in H_{\sigma}$, the function $x \mapsto \sigma(x) \alpha$ from $G$ into $H_{\sigma}$ is continuous. However, since the weak and the strong operator topologies coincide on $\mathcal{U}\left(H_{\sigma}\right)$ then the representation $\sigma$ is continuous if for $\alpha, \beta \in H_{\sigma}$, the mapping $x \mapsto\langle\sigma(x) \alpha, \beta\rangle$ from $G$ into $\mathbb{C}$ is continuous. An intertwining operator for unitary representations $\sigma_{1}: G \rightarrow \mathcal{U}\left(H_{\sigma_{1}}\right)$ and $\sigma_{2}: G \rightarrow \mathcal{U}\left(H_{\sigma_{2}}\right)$ is a bounded linear map $T: H_{\sigma_{1}} \rightarrow H_{\sigma_{2}}$ such that

$$
T \sigma_{1}(x)=\sigma_{2}(x) T, \forall x \in G \text {. }
$$

Let us denote by $\mathcal{C}\left(\sigma_{1}, \sigma_{2}\right)$ the set of all intertwining operators of $\sigma_{1}$ and $\sigma_{2}$. If $\mathcal{C}\left(\sigma_{1}, \sigma_{2}\right)$ is non empty then the representations $\sigma_{1}$ and $\sigma_{2}$ are said to be unitary equivalent.

A subspace $M$ of $H_{\sigma}$ is said to be invariant if $\forall x \in G, \sigma(x) M \subset M$. If $\sigma$ has no invariant subspace except $\{0\}$ and $H_{\sigma}$ then $\sigma$ is said to be irreducible. The set of all equivalent classes of 
unitary irreducible representations of $G$ is called the unitary dual of $G$ and it is denoted by $\widehat{G}$. A locally compact group $G$ admits a left Haar measure, that is a nonzero Radon measure $\mu$ on $G$ such that $\mu(x E)=\mu(E)$ for every Borel set $E$ in $G$ and every $x$ in $G$ and a right Haar measure, that is a nonzero Radon measure $\mu$ on $G$ such that $\mu(E x)=\mu(E)$ for every Borel set $E$ in $G$ and every $x$ in $G$ [8, page 37]. One may decide to consider one or other of these measures. A left Haar measure $\mu$ is unique up to a strictly positive multiplicative constant, that is, if $\nu$ is another left Haar measure then $\nu=a \mu$ where $a$ is a positive constant. Let $\mu$ be a left Haar measure. For $s \in G$, set $\nu(f)=\mu\left(f_{s^{-1}}\right), \forall f \in \mathcal{K}(G)$ where $\mathcal{K}(G)$ designates the space of all compact support functions on $G$ and $f_{s^{-1}}$ the right translated of $f$ by $s$. One can easily verify that $\nu$ is a left Haar measure. Then there exists a unique positive number $\Delta_{G}(s)$ such that $\nu(f)=\Delta_{G}(s) \mu(f)$. This means that

$$
\int_{G} f\left(x s^{-1}\right) d \mu(x)=\Delta_{G}(s) \int_{G} f(x) d \mu(x) .
$$

Let us denote by $\mathbb{R}_{+}^{*}$ the multiplicative group of positive real numbers. The function $\Delta_{G}$ called the modular function of $G$, is a continuous homomorphism from $G$ into $\mathbb{R}_{+}^{*}$, that is $\Delta_{G}$ is continuous and

$$
\forall s_{1}, s_{2} \in G, \Delta_{G}\left(s_{1} s_{2}\right)=\Delta_{G}\left(s_{1}\right) \Delta_{G}\left(s_{2}\right) .
$$

The group $G$ is said to be unimodular if $\Delta_{G} \equiv 1$. Therefore in a unimodular group every left Haar measure is also a right Haar measure. Abelian groups, compact groups and the Heisenberg group are some examples of unimodular groups.

A continuous unitary representation $\sigma$ of a locally compact group $G$ is said to be squareintegrable if for all $\alpha, \beta \in H_{\sigma}$ the following functions $x \mapsto\langle\sigma(x) \alpha, \beta\rangle$, called the coefficients of $\sigma$, are square integrable with respect the left (or right) Haar measure of $G$ [10, page 424]. The set of equivalent classes of square integrable representations is called the discrete series of $G$. The proposition below will be useful in the sequel (see [10]).

Proposition 2.1. Let $G$ be a locally compact unimodular group with left Haar measure $d x$. Let $\sigma$ be a square integrable irreducible representation of $G$. Then there exists a positive constant $d_{\sigma}$ such that

$$
\int_{G}\langle\sigma(x) \alpha, \beta\rangle \overline{\langle\sigma(x) \gamma, \delta\rangle} d x=\frac{\langle\alpha, \gamma\rangle \overline{\langle\beta, \delta\rangle}}{d_{\sigma}} .
$$

The constant $d_{\sigma}$ is called the formal degree of the representation $\sigma$. Proposition 2.1 extends to non-unimodular locally compact groups by the Duflo-Moore theorem [7]. 


\section{Fourier-Stieltjes TRANSFORM}

In this section, we deal with the Fourier-Stieltjes transform of vector measures on locally compact groups. We fix a square integrable irreducible representation $\sigma$ of a locally compact unimodular group $G$. We denote by $H_{\sigma}$ its representation space and by $d_{\sigma}$ its formal degree. Finally we fix a basis $\left(\xi_{\lambda}^{\sigma}\right)_{\lambda \in \Lambda}$ of $H_{\sigma}$. Then the orthogonality relations in Proposition 2.1 become

$$
\int_{G}\left\langle\sigma(x) \xi_{i}^{\sigma}, \xi_{j}^{\sigma}\right\rangle \overline{\left\langle\sigma(x) \xi_{k}^{\sigma}, \xi_{l}^{\sigma}\right\rangle} d x=\frac{\left\langle\xi_{i}^{\sigma}, \xi_{k}^{\sigma}\right\rangle \overline{\left\langle\xi_{j}^{\sigma}, \xi_{l}^{\sigma}\right\rangle}}{d_{\sigma}}=\frac{1}{d_{\sigma}} \delta_{i k} \delta_{j l}
$$

where $i, j, k, l \in \Lambda$.

We also consider a complex Hilbert space $\mathfrak{H}$ and denote the space of compact operators on $\mathfrak{H}$ by $\mathcal{B}_{0}(\mathfrak{H})$. It is well-known that $\mathcal{B}_{0}(\mathfrak{H})$ is a $C^{*}$-algebra. In the sequel one can deal with a general $C^{*}$-algebra $\mathcal{A}$ but from our desire to be concret, we fix $\mathcal{A}=\mathcal{B}_{0}(\mathfrak{H})$.

Proposition 3.1. Let $G$ be a locally compact unimodular group. Let $m$ be a bounded $\mathcal{B}_{0}(\mathfrak{H})$-valued measure on $G$. Then the mapping

$$
\varphi:(\xi, \eta) \mapsto \int_{G}\left\langle\sigma\left(x^{-1}\right) \xi, \eta\right\rangle d m(x)
$$

is a bounded sesquilinear mapping from $H_{\sigma} \times H_{\sigma}$ into $\mathcal{B}_{0}(\mathfrak{H})$.

Proof. The sesquilinearity is trivial. Let us prove the boundedness.

$$
\begin{aligned}
\|\varphi(\xi, \eta)\| & =\left\|\int_{G}\left\langle\sigma\left(x^{-1}\right) \xi, \eta\right\rangle d m(x)\right\| \\
& \leq \int_{G}\left|\left\langle\sigma\left(x^{-1}\right) \xi, \eta\right\rangle\right| d|m|(x) \\
& \leq \int_{G}\left\|\sigma\left(x^{-1}\right) \xi\right\|\|\eta\| d|m|(x) \\
& \leq\|\xi\|\|\eta\|\|m\| .
\end{aligned}
$$

Thus $\varphi$ is bounded and $\|\varphi\| \leq\|m\|$.

Following [3] where authors dealt with the Fourier-Stieltjes transform on compact groups we set the following definition.

Definition 3.1. The $\sigma$-Fourier-Stieltjes transform of a bounded $\mathcal{B}_{0}(\mathfrak{H})$-valued measure $m$ on a locally compact unimodular group $G$ is the $\mathcal{B}_{0}(\mathfrak{H})$-valued bounded sesquilinear map $\mathcal{F}^{\sigma}$ m on $H_{\sigma} \times H_{\sigma}$ defined by

$$
\mathcal{F}^{\sigma} m(\xi, \eta)=\int_{G}\left\langle\sigma\left(x^{-1}\right) \xi, \eta\right\rangle d m(x), \quad \xi, \eta \in H_{\sigma}
$$


The $\sigma$-Fourier transform of a strong Bochner integrable $\mathcal{B}_{0}(\mathfrak{H})$-valued function $f$ on $G$ (with respect to the Haar measure of $G$ ) is defined by

$$
\mathcal{F}^{\sigma} f(\xi, \eta)=\int_{G}\left\langle\sigma\left(x^{-1}\right) \xi, \eta\right\rangle f(x) d x, \xi, \eta \in H_{\sigma}
$$

Now we want to move from sesquilinear mappings to linear operators by the means of tensor product spaces [14]. Let us denote by $\mathcal{S}\left(H_{\sigma} \times H_{\sigma}, \mathcal{B}_{0}(\mathfrak{H})\right)$ the set of bounded sesquilinear maps from $H_{\sigma} \times H_{\sigma}$ into $\mathfrak{B}_{0}(\mathfrak{H})$. We denote by $\bar{H}_{\sigma}$ the conjugate Hilbert space of $H_{\sigma}$ and by $H_{\sigma} \widehat{\otimes}_{\pi} \bar{H}_{\sigma}$ the completion of $H_{\sigma} \otimes \bar{H}_{\sigma}$ under the projective tensor product norm. Let us denote by $\mathcal{B}\left(H_{\sigma} \widehat{\otimes}_{\pi} \bar{H}_{\sigma}, \mathcal{B}_{0}(\mathfrak{H})\right)$ the space of bounded linear operators from $H_{\sigma} \widehat{\otimes}_{\pi} \bar{H}_{\sigma}$ into $\mathcal{B}_{0}(\mathfrak{H})$. Then we have the identification $\mathcal{S}\left(H_{\sigma} \times H_{\sigma}, \mathcal{B}_{0}(\mathfrak{H})\right) \simeq \mathcal{B}\left(H_{\sigma} \widehat{\otimes}_{\pi} \bar{H}_{\sigma}, \mathcal{B}_{0}(\mathfrak{H})\right)$ [13]. Therefore one can linearize the transformed measure $\mathcal{F}^{\sigma} m$ by viewing it as the element of the space $\mathcal{B}\left(H_{\sigma} \widehat{\otimes}_{\pi} \bar{H}_{\sigma}, \mathcal{B}_{0}(\mathfrak{H})\right)$ defined by

$$
\mathcal{F}^{\sigma} m(\xi \otimes \eta)=\int_{G}\left\langle\sigma\left(x^{-1}\right) \xi, \eta\right\rangle d m(x), \xi \in H_{\sigma}, \eta \in \bar{H}_{\sigma} .
$$

In the same way, the transformed of a function $f \in L^{1}\left(G, \mathcal{B}_{0}(\mathfrak{H})\right)$ is given by

$$
\mathcal{F}^{\sigma} f(\xi \otimes \eta)=\int_{G}\left\langle\sigma\left(x^{-1}\right) \xi, \eta\right\rangle f(x) d x
$$

A little computation as in the proof of Proposition 3.1 shows that $\mathcal{F}^{\sigma} m$ and $\mathcal{F}^{\sigma} f$ are bounded operators from $H_{\sigma} \otimes \bar{H}_{\sigma}$ into $\mathcal{B}_{0}(\mathfrak{H})$ with

$$
\left\|\mathcal{F}^{\sigma} m\right\| \leq\|m\| \text { and }\left\|\mathcal{F}^{\sigma} f\right\| \leq\|f\|_{L^{1}}
$$

By the density of $H_{\sigma} \otimes \bar{H}_{\sigma}$ in $H_{\sigma} \widehat{\otimes}_{\pi} \bar{H}_{\sigma}$ the operators $\mathcal{F}^{\sigma} m$ and $\mathcal{F}^{\sigma} f$ are uniquely extended to bounded operators from $H_{\sigma} \widehat{\otimes}_{\pi} \bar{H}_{\sigma}$ into $\mathcal{B}_{0}(\mathfrak{H})$.

For $i, j \in \Lambda$ and $x \in G$ set

$$
u_{i j}^{\sigma}(x)=\left\langle\sigma(x) \xi_{i}^{\sigma}, \xi_{i}^{\sigma}\right\rangle
$$

Proposition 3.2. Let $G$ be a locally compact unimodular group and let $\sigma$ be a square integrable irreducible representation of $G$. Then

$$
\mathcal{F}^{\sigma} u_{i j}^{\sigma}\left(\xi_{k}^{\sigma} \otimes \xi_{l}^{\sigma}\right)=\frac{\delta_{i l} \delta_{j k}}{d_{\sigma}}
$$




\section{Proof.}

$$
\begin{aligned}
\mathcal{F}^{\sigma} u_{i j}^{\sigma}\left(\xi_{k}^{\sigma} \otimes \xi_{l}^{\sigma}\right) & =\int_{G}\left\langle\sigma\left(x^{-1}\right) \xi_{k}^{\sigma}, \xi_{l}^{\sigma}\right\rangle\left\langle\sigma(x) \xi_{i}^{\sigma}, \xi_{j}^{\sigma}\right\rangle d x \\
& =\int_{G}\left\langle\sigma(x) \xi_{i}^{\sigma}, \xi_{j}^{\sigma}\right\rangle \overline{\left\langle\sigma(x) \xi_{l}^{\sigma}, \xi_{k}^{\sigma}\right\rangle} d x \\
& =\frac{\left\langle\xi_{i}^{\sigma}, \xi_{l}^{\sigma}\right\rangle \overline{\left\langle\xi_{j}^{\sigma}, \xi_{k}^{\sigma}\right\rangle}}{d_{\sigma}} \\
& =\frac{\delta_{i l} \delta_{j k}}{d_{\sigma}}
\end{aligned}
$$

Proposition 3.3. Let $G$ be a locally compact unimodular group and let $\sigma$ be a square integrable irreducible representation of $G$. Consider the vectors $X=\sum_{k \in K} \alpha_{k} \xi_{k}^{\sigma}$ and $Y=\sum_{l \in L} \beta_{l} \xi_{l}^{\sigma}$ where $K$ and $L$ are finite subsets of $\Lambda$. Then

$$
\mathcal{F}^{\sigma} u_{i j}^{\sigma}(X \otimes Y)=\frac{\alpha_{j} \overline{\beta_{i}}}{d_{\sigma}}
$$

\section{Proof.}

$$
\begin{aligned}
\mathcal{F}^{\sigma} u_{i j}^{\sigma}(X \otimes Y) & =\int_{G}\left\langle\sigma\left(x^{-1}\right) X, Y\right\rangle\left\langle\sigma(x) \xi_{i}^{\sigma}, \xi_{j}^{\sigma}\right\rangle d x \\
& =\int_{G}\left\langle\sigma\left(x^{-1}\right) \sum_{k \in K} \alpha_{k} \xi_{k}^{\sigma}, \sum_{l \in L} \beta_{l} \xi_{l}^{\sigma}\right\rangle\left\langle\sigma(x) \xi_{i}^{\sigma}, \xi_{j}^{\sigma}\right\rangle d x \\
& =\int_{G} \sum_{k \in K} \sum_{l \in L} \alpha_{k} \overline{\beta_{l}}\left\langle\sigma\left(x^{-1}\right) \xi_{k}^{\sigma}, \xi_{l}^{\sigma}\right\rangle\left\langle\sigma(x) \xi_{i}^{\sigma}, \xi_{j}^{\sigma}\right\rangle d x \\
& =\int_{G} \sum_{k \in K} \sum_{l \in L} \alpha_{k} \overline{\beta_{l}} u_{k l}^{\sigma}\left(x^{-1}\right) u_{i j}^{\sigma}(x) d x \\
& =\sum_{k \in K} \sum_{l \in L} \alpha_{k} \overline{\beta_{l}} \int_{G} u_{k l}^{\sigma}\left(x^{-1}\right) u_{i j}^{\sigma}(x) d x \\
& =\sum_{k \in K} \sum_{l \in L} \alpha_{k} \overline{\beta_{l}} \int_{G}\left\langle\sigma(x) \xi_{i}^{\sigma}, \xi_{j}^{\sigma}\right\rangle \overline{\left\langle\sigma(x) \xi_{l}^{\sigma}, \xi_{k}^{\sigma}\right\rangle} d x \\
& =\sum_{k \in K} \sum_{l \in L} \alpha_{k} \overline{\beta_{l}} \frac{\left\langle\xi_{i}^{\sigma}, \xi_{l}^{\sigma}\right\rangle \overline{\left\langle\xi_{j}^{\sigma}, \xi_{k}^{\sigma}\right\rangle}}{d_{\sigma}} \\
& =\frac{\alpha_{j} \overline{\beta_{i}}}{d_{\sigma}} .
\end{aligned}
$$


Proposition 3.4. Let $m$ be a bounded $\mathcal{B}_{0}(\mathfrak{H})$-valued measure on a locally compact unimodular group $G$. Let $\sigma$ be a square integrable irreducible representation of $G$. Then there exists a family $\left(T_{k l}\right)_{k \in K, l \in L}$ of elements of $\mathcal{B}_{0}(\mathfrak{H})$ such that

$$
\mathcal{F}^{\sigma} m=d_{\sigma} \sum_{k \in K} \sum_{l \in L} T_{k l} \mathcal{F}^{\sigma} u_{k l}^{\sigma} .
$$

Proof. Let $X=\sum_{k \in K} \alpha_{k} \xi_{k}^{\sigma}$ and $Y=\sum_{l \in L} \beta_{l} \xi_{l}^{\sigma}$. Then we have

$$
\begin{aligned}
\mathcal{F}^{\sigma} m(X \otimes Y) & =\int_{G}\left\langle\sigma\left(x^{-1}\right) \sum_{k \in K} \alpha_{k} \xi_{k}^{\sigma}, \sum_{l \in L} \beta_{l} \xi_{l}^{\sigma}\right\rangle d m(x) \\
& =\sum_{k \in K} \sum_{l \in L} \alpha_{k} \overline{\beta_{l}} \mathcal{F}^{\sigma} m\left(\xi_{k}^{\sigma} \otimes \xi_{l}^{\sigma}\right) \\
& =d_{\sigma} \sum_{k \in K} \sum_{l \in L} T_{k l} \mathcal{F}^{\sigma} u_{k l}^{\sigma}(X \otimes Y) \text { (use Proposition 3.3) }
\end{aligned}
$$

where we have set $T_{k l}=\mathcal{F}^{\sigma} m\left(\xi_{k}^{\sigma} \otimes \xi_{l}^{\sigma}\right)$. Therefore $\mathcal{F}^{\sigma} m=d_{\sigma} \sum_{k \in K} \sum_{l \in L} T_{k l} \mathcal{F}^{\sigma} u_{k l}^{\sigma}$.

We denote by $\mathcal{B}^{\sigma}\left(G, \mathcal{B}_{0}(\mathfrak{H})\right)$ the set of functions $f$ defined on $G$ by

$$
f(x)=\sum_{k \in \Lambda} \sum_{l \in \Lambda} T_{k l} u_{k l}^{\sigma}(x)
$$

where the $T_{i j}$ are compact operators on $\mathfrak{H}$.

Proposition 3.5. Let $G$ be a locally compact unimodular group. Let $\sigma$ be a square integrable irreducible representation of $G$. If $T \in \mathcal{B}\left(H_{\sigma} \widehat{\otimes}_{\pi} \bar{H}_{\sigma}, \mathcal{B}_{0}(\mathfrak{H})\right)$ then there exists $f \in \mathcal{B}^{\sigma}\left(G, \mathcal{B}_{0}(\mathfrak{H})\right)$ such that $\mathcal{F}^{\sigma} f=T$.

Proof. Let $T$ be an element of $\mathcal{B}\left(H_{\sigma} \widehat{\otimes}_{\pi} \bar{H}_{\sigma}, \mathcal{B}_{0}(\mathfrak{H})\right)$. Set $T_{k l}=d_{\sigma} T\left(\xi_{l}^{\sigma} \otimes \xi_{k}^{\sigma}\right), k, l \in \Lambda$. Then the function $f(\cdot)=\sum_{k \in \Lambda} \sum_{l \in \Lambda} T_{k l} u_{k l}^{\sigma}(\cdot)$ belongs to $\mathcal{B}^{\sigma}\left(G, \mathcal{B}_{0}(\mathfrak{H})\right)$. Moreover

$$
\begin{aligned}
\mathcal{F}^{\sigma} f\left(\xi_{i}^{\sigma} \otimes \xi_{j}^{\sigma}\right) & =\sum_{k \in \Lambda} \sum_{l \in \Lambda} T_{k l} \mathcal{F}^{\sigma} u_{k l}^{\sigma}\left(\xi_{i}^{\sigma} \otimes \xi_{j}^{\sigma}\right) \\
& =\sum_{k \in \Lambda} \sum_{l \in \Lambda} \frac{\delta_{k j} \delta_{l i}}{d_{\sigma}} T_{k l} \quad \text { (use Proposition 3.2) } \\
& =\frac{1}{d_{\sigma}} T_{j i} \\
& =T\left(\xi_{i}^{\sigma} \otimes \xi_{j}^{\sigma}\right) .
\end{aligned}
$$

Hence $\mathcal{F}^{\sigma} f=T$.

We derive from the above proposition the following consequence. 
Corollary 3.1. Let $G$ be a locally compact unimodular group. Let $\sigma$ be a square integrable irreducible representation of $G$. If $T \in \mathcal{B}\left(H_{\sigma} \widehat{\otimes}_{\pi} \bar{H}_{\sigma}, \mathcal{B}_{0}(\mathfrak{H})\right)$ then the following integral representation of $T$ holds:

$$
T\left(\xi_{i}^{\sigma} \otimes \xi_{j}^{\sigma}\right)=\sum_{k \in \Lambda} \sum_{l \in \Lambda} T_{k l} \int_{G}\left\langle\sigma\left(x^{-1}\right) \xi_{i}^{\sigma}, \xi_{j}^{\sigma}\right\rangle\left\langle\sigma(x) \xi_{k}^{\sigma}, \xi_{l}^{\sigma}\right\rangle d x
$$

where the $T_{k l}$ are compact operators on $\mathfrak{H}$.

\section{REFERENCES}

[1] S. T. Ali, J.-P. Antoine, J.-P. Gazeau, Coherent States, Wavelets and their Generalizations, Springer-Verlag, New York, 1999.

[2] P. Aniello, Square Integrable Representations, An Invaluable Tool, In: Antoine JP., Bagarello F., Gazeau JP. (eds) Coherent States and Their Applications. Springer Proceedings in Physics, vol 205. Springer, Cham, 2018.

[3] V.S.K. Assiamoua, A. Olubummo, Fourier-Stieltjes transforms of vector-valued measures on compact groups, Acta Sci. Math. 53 (1989), 301-307.

[4] W. Averson, An Invitation to $C^{*}$-algebras, Springer-Verlag, New York, 1976.

[5] A. Deitmar, S. Echterhoff, Principles of Harmonic Analysis, Springer, Switzerland, 2014.

[6] J. Diestel, J. J. Uhl, Jr, Vector Measures, Amer. Math. Soc., 15, Providence, Rhode Island, 1977.

[7] M. Duflo, C. C. Moore, On the regular representation of a nonunimodular locally compact group, J. Funct. Anal. 21 (1976), 209-243.

[8] G.B. Folland, A Course in Abstract Harmonic Analysis, CRC Press, Boca Raton, 1995.

[9] H. Führ, Abstract Harmonoc Analysis of Continuous Wavelet Transforms, Lectures notes in Mathematics 1863, Springer-Verlag Berlin Heidelberg, 2005.

[10] S.A. Gaal, Linear Analysis and Representation Theory, Springer-Verlag New York Heidelberg Berlin, 1973.

[11] K. Gbemou and Y. Mensah, Aspects of the Fourier-Stieltjes transform of $C^{*}$-algebra valued measures, J. Adv. Math. Computer Sci. 33 (5) (2019), AMCS.50893.

[12] E. Kaniuth, A. T.-M. Lau, Fourier and Fourier-Stieltjes algebras on locally compact groups, Mathematical Surveys and Monographs, 231, Amer. Math. Soc., Providence, Rhode Island, 2018.

[13] Y. Mensah, Facts about the Fourier-Stieltjes transform of vector measures on compact groups, Int. J. Anal. Appl. 2 (1) (2013), 19-25.

[14] R.A. Ryan, Introduction to Tensor Products of Banach Spaces, Springer, London, 2002.

[15] K. Wodome, Y. Mensah, On a transform of Fourier-Stieltjes type for $C^{*}$-algebra valued measures, Pure Math. Sci. 6 (1) (2017), 113-121. 